\title{
Singularity in classical and quantum Kepler Problem with Weak Anisotropy
}

\author{
Zai-Qiao Bai and Wei-Mou Zheng \\ Institute of Theoretical Physics, Academia Sinica, Beijing 100080, China
}

\begin{abstract}
Anisotropic Kepler problem is investigated by perturbation method in both classical and quantum mechanics. In classical mechanics, due to the singularity of the potential, global diffusion in phase space occurs at an arbitrarily small perturbation parameter. In quantum mechanics, the singularity induces a large transition amplitude between quasi degenerate eigen states, which generically decays as $\hbar$ in the semi-classical limit.
\end{abstract}

PACS number: 03.20.+i; 03.65.-w; 03.65.sq

Keywords: Anisotropic Kepler problem, Singularity, Quantum-classical correspondence 
The anisotropic Kelper problem (AKP) is a 2-dimensional Hamiltonian system defined by

$$
H=\frac{1}{2}\left(p_{x}^{2}+p_{y}^{2}\right)-\frac{1}{\sqrt{\nu x^{2}+\mu y^{2}}}
$$

with $\mu>\nu>0$ and $\mu \nu=1$ (For a detailed discussion of its dynamics, see [1] ). While the system reduces to the Kepler problem when $\mu=1$, the existence of chaos was rigorously proved by Gutzwiller and Devaney in the case of $\mu / \nu>9 / 8$ [2, 3]. The emergence of irregular dynamics dose not follow the conventional KAM scenario due to the singularity at the origin. In fact, the extremely unstable motion in the vicinity of the origin played a central role in Gutzwiller and Devaney's proof. The dynamical implication of the singularity when $\mu \rightarrow 1$ is, however, still not clear. When $\mu \approx 1$, (1) can be rewritten as

$$
H=\frac{1}{2}\left(p_{x}^{2}+p_{y}^{2}\right)-\frac{1}{r}-\epsilon \frac{\cos 2 \theta}{r}=H_{0}+\epsilon F
$$

where $r=\sqrt{x^{2}+y^{2}}$ and $\theta=\tan ^{-1}(y / x)$ while $\epsilon=(\mu-1) / 2$ appears as a natural perturbation parameter. In this letter we shall study the classical and quantum AKP by perturbation method. We hope this will provide a deeper understanding of the quantum-classic correspondence in nonKAM system.

We begin with the classical mechanics. Kepler problem is a maximally integrable system. All its orbits are closed with period $T=2 \pi(-2 E)^{-3 / 2}$, where $E=H_{0}<0$. This global periodic motion will be destroyed by an infinitely small perturbation. The first order effect of a slight perturbation can be described by the drift of closed orbit, i.e., the slow variation of its parameters. Therefore, at this level of approximation, we can consider the motion in the space of all closed Kepler orbits (orbital space ).

The orbital space can be conveniently constructed by taking advantage of the so(3) dynamical symmetry. Let

$$
J_{1}=\left(\cos \theta-p_{y} J_{3}\right) / \sqrt{-2 E}, \quad J_{2}=\left(\sin \theta+p_{x} J_{3}\right) / \sqrt{-2 E}, \quad \text { and } \quad J_{3}=x p_{y}-y p_{x}
$$

$\left(J_{1}, J_{2}, J_{3}\right)$ form a $s o(3)$ algebra, i.e., $\left\{J_{i}, J_{j}\right\}=\varepsilon_{i j k} J_{k}, i, j=1,2,3$ and $H_{0}=\frac{-1}{2\left(J_{1}^{2}+J_{2}^{2}+J_{3}^{2}\right)}$. There is a one-to-one correspondence between $\mathcal{J}=\left(J_{1}, J_{2}, J_{3}\right)$ and close Kepler orbits. Specif- 
ically, write $\left(J_{1}, J_{2}, J_{3}\right)=\frac{1}{\sqrt{-2 E}}(\sin \alpha \cos \beta, \sin \alpha \sin \beta, \cos \alpha)$, the corresponding Kepler orbit in configuration space is defined as

$$
r=\frac{J_{3}^{2}}{1-\sin \alpha \cos (\theta-\beta)}
$$

while the sign of $J_{3}$ determines its direction. Therefore, the orbital space is coordinated by $\left(J_{1}, J_{2}, J_{3}\right)($ or $(E, \alpha, \beta))$.

The variation of $J_{i}$ in one Kepler period is given by

$$
J_{i}(t+T)=J_{i}(t)+\epsilon T\left\{J_{i}, F_{0}(\mathcal{J})\right\}+o(\epsilon), \quad i=1,2,3,
$$

where $F_{0}$ is the time average of $F$ over closed Kepler orbit,

$$
F_{0}=F_{0}(\mathcal{J})=\frac{1}{T} \int_{0}^{2 \pi} F(r, \theta) \frac{r^{2}}{\left|J_{3}\right|} d \theta=2 E \frac{1-|\cos \alpha|}{1+|\cos \alpha|} \cos 2 \beta .
$$

When $\epsilon \rightarrow 0$, Eq. (5) can be approximated by a differential equation,

$$
\frac{d J_{i}}{d t}=\epsilon\left\{J_{i}, F_{0}(\mathcal{J})\right\}, \quad i=1,2,3 .
$$

$\left\{H_{0}, F_{0}\right\}=0$ implies that the motion in the orbital space is confined within a sphere $\mathcal{S}_{E}$ with $H_{0}=E=$ const.. Restricting so(3) Poisson structure on $\mathcal{S}_{E}$ induces a natural sympletic form $\omega_{2}=\frac{\sin \alpha}{\sqrt{-2 E}} d \beta \wedge d \alpha$, which is, up to a constant, the ordinary area element.

As a two-dimensional Hamiltonian system, the dynamics on $\mathcal{S}_{E}$ can be easily determined by the contour chart of the effective Hamiltonian $F_{0}$. There exist six fixed points on the sphere. The poles $(\alpha=0, \pi)$, which represent the two circular Kepler orbits, are unstable while the four on equator $(\alpha=\pi / 2, \beta=0, \pm \pi / 2, \pi)$, which corresponds to the linear orbits on the $x$ or $y$ axis, are stable. The remaining orbits are either the heteroclinic orbits $(\beta= \pm \pi / 4, \pm 3 \pi / 4)$ that connect the poles or periodic orbits surrounding one of the stable fixed points. In other words, besides separatrixs, the classical motion consists of four islands centered respectively at the degenerate Kepler orbits on the $x$ and $y$ axis.

It should be pointed out that the simple picture given by perturbation analysis is not correct at the vicinity of $\alpha=\pi / 2$, i.e., the collision orbits, where $\epsilon F$ is not bounded. In fact, the first 
order derivative of $F_{0}$ is in general not continuous at equator. This non-smoothness manifests the non-perturbative nature of the motion near the origin. Noticing that all orbits on $\mathcal{S}_{E}$ except for the poles cross $\alpha=\pi / 2$, we conclude that the global dynamics of AKP cannot be described by perturbation with respect to Kepler problem even at the limit $\epsilon \rightarrow 0$. Our numerical study show that the collision orbits provide a passage-way for global diffusion in the orbital (and hence phase) space (Fig. 1).

Now we turn to quantum mechanics. The so(3) symmetry of quantum planar Kepler problem is constructed in a way similar to its classical analog. Specifically,

$$
\left\{\begin{array}{l}
J_{1}=\left[\cos \theta-\frac{1}{2}\left(p_{y} J_{3}+J_{3} p_{y}\right)\right] / \sqrt{-2 H_{0}} \\
J_{2}=\left[\sin \theta+\frac{1}{2}\left(p_{x} J_{3}+J_{3} p_{x}\right)\right] / \sqrt{-2 H_{0}} \\
J_{3}=x p_{y}-y p_{x}
\end{array}\right.
$$

$\left[J_{j}, J_{k}\right]=i \varepsilon_{j k l} J_{l}$ and $H_{0}=\frac{-1}{2\left(J^{2}+\frac{1}{4}\right)}(\hbar \equiv 1)$. Let $\{|n, m>: m \leq| n \mid, n=0,1, \ldots\}$ be the standard so(3) orthonormal set, i.e., $J_{3}|n, m>=m| n, m>$ and $J^{2}|n, m>=n(n+1)| n, m>$. $\mid n, m>$ is an eigenstate of $H_{0}$ with energy $E=E_{n}=\frac{-1}{2\left(n+\frac{1}{2}\right)^{2}}$ and wave function in coordinate representation given by

$$
\Psi_{n, m}(r, \theta)=(-1)^{m} \frac{1}{|2 m| !} \sqrt{\frac{(n+|m|) !}{(n-|m|) !}} \frac{4 s^{|m|}}{(2 n+1)^{3 / 2}} e^{-\frac{1}{2} s} F(-n+|m|, 2|m|+1, s) \frac{e^{i m \theta}}{\sqrt{2 \pi}}
$$

where $s=4 r /(2 n+1)$ and $F(a, b, x)$ is the confluent hypergeometric function.

The first order effect of a slight perturbation is the mixing of states with definite $n$. In the interaction representation, the long-time evolution when $\epsilon \rightarrow 0$ is given by an effective Shördinger equation

$$
i \frac{d}{d \tau} \Psi=\bar{F} \Psi
$$

where $\bar{F}$ is the restriction of $F$ in eigen spaces of $H_{0}$, i.e.,

$$
<n^{\prime}, m^{\prime}|\bar{F}| n, m>=\delta_{n, n^{\prime}}<n^{\prime}, m^{\prime}|F| n, m>\text {. }
$$

Eq. (10) can be regarded as the quantum counterpart of Eq. (6) and hence $F_{0}$ is the classical 
correspondence of $\bar{F}$. The matrix elements of $\bar{F}$ is readily evaluated in coordinate representation,

$$
<n, m^{\prime}|\bar{F}| n, m>=E_{n} \sqrt{\frac{\left(n+m_{1}\right) !\left(n-m_{1}\right) !}{\left(n+m_{2}\right) !\left(n-m_{2}\right) !}} \delta_{\left|m^{\prime}-m\right|, 2},
$$

where $m_{1}=\min \left\{|m|,\left|m^{\prime}\right|\right\}$ and $m_{2}=\max \left\{|m|,\left|m^{\prime}\right|\right\}$.

By diagonalizing $\bar{F}$ in each $2 n+1$-dimensional subspace, we can study the classical-quantum correspondence in the framework of perturbation theory. Notice that $\exp \left(i \frac{\pi}{2} J_{3}\right) \bar{F}+\bar{F} \exp \left(i \frac{\pi}{2} J_{3}\right)=$ 0 , the spectrum of $\bar{F}$ is symmetric with respect to 0 . We shall focus on the positive part, which corresponds to the classical islands centered at fixed points $(\alpha, \beta)=(\pi / 2, \pi / 2)$ and $(\pi / 2,3 \pi / 2)$.

The classical orbits centered at fixed point $(\pi / 2, \pi / 2)$ contribute to the spectrum of $\bar{F}$ according to the semi-classical quantization rule,

$$
\frac{1}{2 \pi \hbar} \int_{\sigma\left(\lambda_{k}\right)} \omega_{2}=k+\mu_{s} / 4, \quad k=0,1, \ldots
$$

where $\sigma(\lambda) \subset S_{E}$ is the region enclosed by orbit with $F_{0}(E, \alpha, \beta)=\lambda$ and the Maslov index $\mu_{s}=2$. For convenience, we rescale $E=-\frac{1}{2}$ and $\hbar=\frac{2}{2 n+1}$ so that $\left|F_{0}\right| \leq 1$ and Eq. (13) yields

$$
S\left(n, \lambda_{k}\right) \equiv \frac{n+\frac{1}{2}}{\pi}\left(\cos ^{-1} \lambda_{k}+\frac{2 \lambda_{k} \ln \lambda_{k}}{\sqrt{1-\lambda_{k}^{2}}}\right)=k+\frac{1}{2} .
$$

The orbits surrounding point $(\pi / 2,3 \pi / 2)$ gives the same contribution so that the semi-classical spectrum consists of 2-fold degeneracies. Eq. (14) is a good approximation of the exact spectrum (Table 1).

The degeneracy predicted by the semi-classical rule is not exact due to quantum tunneling between the two quasi static states each of them classically corresponds to one periodic orbit. The splitting of spectrum $(\Delta \lambda)$ is connected with the transition amplitude accumulated in one Kepler period $\left(A_{T}\right)$ by $A_{T}=\epsilon \Delta \lambda T / 2 \hbar$. In conventional quantum system, such as a particle confined in double-well potential, $\Delta \lambda$ decays as $\sim \hbar^{\gamma} \exp (-S / \hbar)$ when $\hbar \rightarrow 0$, which vanishes faster than any power of $\hbar$. Fig. 2 shows $\Delta \lambda_{s c} \equiv \Delta \lambda / \hbar^{2}$ for $n=100$ and 1000, from which we can see that $\Delta \lambda \sim \hbar^{2}$ (or $A_{T} \sim \hbar$ ) in the semi-classical limit. As a characteristic phenomenon 
in in non-smooth system[4], this power-law reflects the non-smoothness in $F_{0}$ and hence the singularity in AKP.

In summary, we have studied in this letter the Anisotropic Kepler problem by perturbation method. By using the dynamical symmetry of the unperturbed system, we obtained an effective Hamiltonian in both classical and quantum mechanics. We shown that the long-time evolution of most of the classical orbits is non-perturbative due to the singularity at origin and global diffusion in phase space occurs even at an arbitrarily small perturbation parameter. In quantum mechanics, we shown that the characteristic phenomenon attributed the singularity is the the power-law $\hbar$-dependence of tunneling amplitude between states which classically correspond to separated periodic orbits.

\section{References}

[1] M. C. Gutzwiller, Chaos in Classical and Quantum Mechanics (Springer, New York, 1990).

[2] M. C. Gutzwiller, J. Math. Phys. 18, 806 (1977).

[3] R. L. Devaney, Invent. Math. 45, 221 (1978).

[4] Z. Q. Bai preprint (quant-ph/0110126) 
Table

\begin{tabular}{|c|c|c|c|c|}
\hline$k$ & $\lambda$ & $S(n, \lambda)$ & $\lambda$ & $S(n, \lambda)$ \\
\hline 0 & 0.6883 & 0.34 & 0.6512 & 0.42 \\
1 & 0.3335 & 1.52 & 0.3264 & 1.55 \\
2 & 0.1944 & 2.43 & 0.1875 & 2.48 \\
3 & 0.0898 & 3.50 & 0.0856 & 3.55 \\
4 & 0.0307 & 4.43 & 0.0293 & 4.46 \\
\hline
\end{tabular}

Tab.1 Spectrum of $\bar{F}$ at $n=10$. 

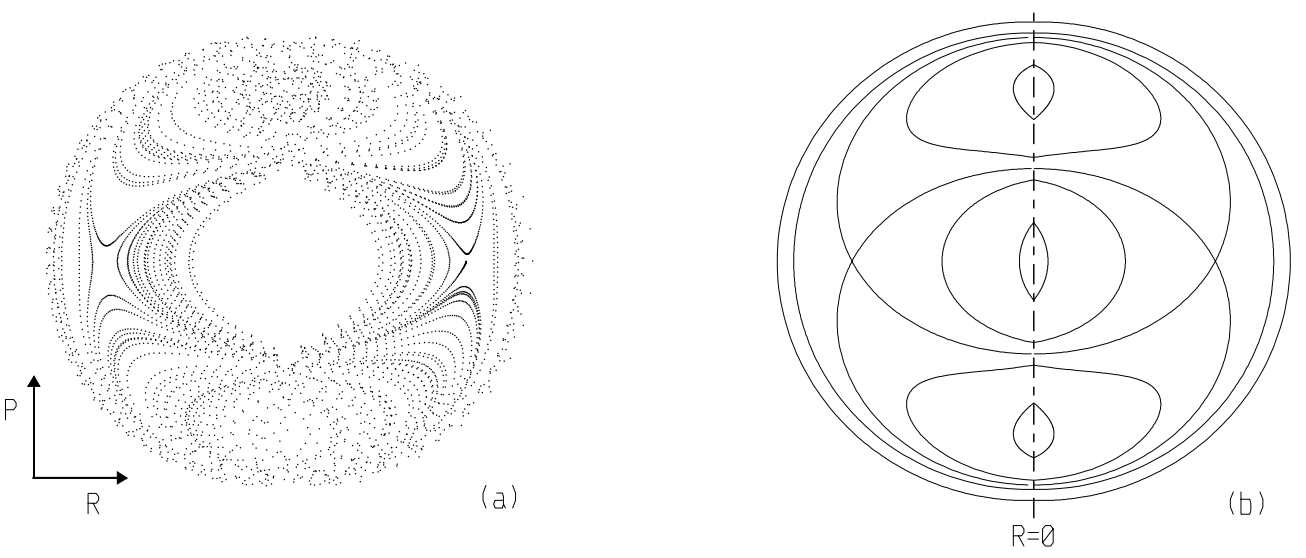

Figure 1: (a) Numerical calculation of one orbit at $\mu=1.04 .5000$ intersections are plotted on the Poincaré surface of section defined at $\theta=0$. with coordinate $(R, P)=\operatorname{sign}\left(p_{y}\right)\left(\sqrt{r}, \sqrt{r} p_{x}\right)$, comparing with the regular structure implied by the first-order perturbation (b).

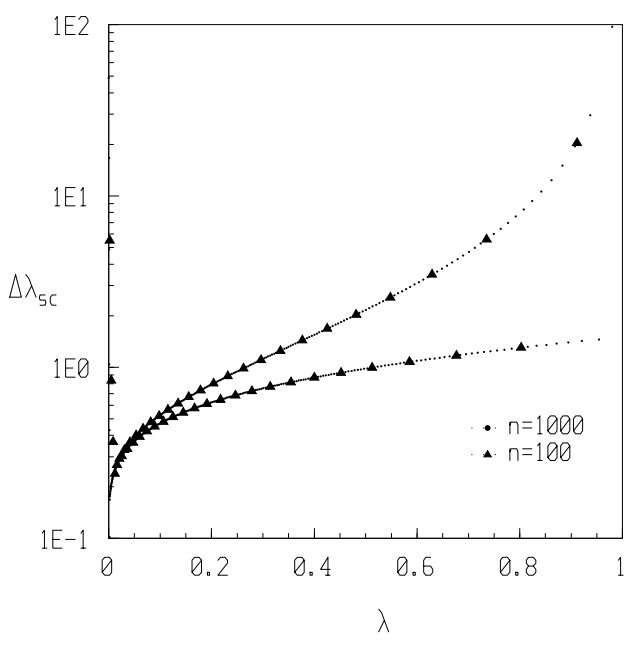

Figure 2: Scaled spectrum splitting $\left(\Delta \lambda_{s c}\right)$ for $n=100$ and 1000. Except for few points near $\lambda=0,\left(\lambda, \Delta \lambda_{s c}\right)$ is approximately located at two curves (determined by whether $k$ is even or odd) irrespective of $n$. 